\title{
Blood pressure thresholds in pregnancy for identifying maternal and infant risk: A secondary analysis of community-level interventions for pre-eclampsia (CLIP) trial data
}

\author{
Jeffrey N. Bone \\ University of British Columbia, Vancouver, Canada \\ Laura A. Magee \\ University of British Columbia, Vancouver, Canada \\ Joel Singer \\ University of British Columbia, Vancouver, Canada \\ Hannah Nathan \\ King's College London, London, UK. \\ Rahat Qureshi \\ Aga Khan University, rahat.qureshi@aku.edu
}

See next page for additional authors

Follow this and additional works at: https://ecommons.aku.edu/coe-wch

Part of the Cardiology Commons, Maternal and Child Health Commons, Obstetrics and Gynecology Commons, and the Women's Health Commons

\section{Recommended Citation}

Bone, J. N., Magee, L. A., Singer, J., Nathan, H., Qureshi, R., Sacoor, C., Sevene, E., Shennan, A., Bellad, M. B., Bhutta, Z. A. (2021). Blood pressure thresholds in pregnancy for identifying maternal and infant risk: A secondary analysis of community-level interventions for pre-eclampsia (CLIP) trial data. The Lancet Global Health, 9(8), e1119-e1128.

Available at: https://ecommons.aku.edu/coe-wch/24 


\section{Authors}

Jeffrey N. Bone, Laura A. Magee, Joel Singer, Hannah Nathan, Rahat Qureshi, Charfudin Sacoor, Esperança Sevene, Andrew Shennan, Mrutyunjaya B. Bellad, and Zulfiqar Ahmed Bhutta 


\section{Blood pressure thresholds in pregnancy for identifying maternal and infant risk: a secondary analysis of Community-Level Interventions for Pre-eclampsia (CLIP) trial data}

Jeffrey N Bone*, Laura A Magee*, Joel Singer, Hannah Nathan, Rahat N Qureshi, Charfudin Sacoor, Esperança Sevene, Andrew Shennan, Mrutyunjaya B Bellad, Shivaprasad S Goudar, Ashalata A Mallapur, Khátia Munguambe, Marianne Vidler, Zulfiqar A Bhutta, Peter von Dadelszen, on behalf of the CLIP study group ${ }^{+}$

\section{Summary}

Background Blood pressure measurement is a marker of antenatal care quality. In well resourced settings, lower blood pressure cutoffs for hypertension are associated with adverse pregnancy outcomes. We aimed to study the associations between blood pressure thresholds and adverse outcomes and the diagnostic test properties of these blood pressure cutoffs in low-resource settings.

Methods We did a secondary analysis of data from 22 intervention clusters in the Community-Level Interventions for Pre-eclampsia (CLIP) cluster randomised trials (NCT01911494) in India (n=6), Mozambique (n=6), and Pakistan $(n=10)$. We included pregnant women aged 15-49 years (12-49 years in Mozambique), identified in their community by trained community health workers, who had data on blood pressure measurements and outcomes. The trial was unmasked. Maximum blood pressure was categorised as: normal blood pressure (systolic blood pressure [sBP] $<120 \mathrm{~mm} \mathrm{Hg}$ and diastolic blood pressure $[\mathrm{dBP}]<80 \mathrm{~mm} \mathrm{Hg}$ ), elevated blood pressure (sBP 120-129 $\mathrm{mm} \mathrm{Hg}$ and $\mathrm{dBP}<80 \mathrm{~mm} \mathrm{Hg}$ ), stage 1 hypertension (sBP 130-139 mm Hg or dBP 80-89 mm Hg, or both), non-severe stage 2 hypertension (sBP 140-159 mm Hg or dBP 90-109 mm Hg, or both), or severe stage 2 hypertension (sBP $\geq 160 \mathrm{~mm} \mathrm{Hg}$ or $\mathrm{dBP} \geq 110 \mathrm{~mm} \mathrm{Hg}$, or both). We classified women according to the maximum blood pressure category reached across all visits for the primary analyses. The primary outcome was a maternal, fetal, or neonatal mortality or morbidity composite. We estimated dose-response relationships between blood pressure category and adverse outcomes, as well as diagnostic test properties.

Findings Between Nov 1, 2014, and Feb 28, 2017, 21069 women (6067 in India, 4163 in Mozambique, and 10839 in Pakistan) contributed 103679 blood pressure measurements across the three CLIP trials. Only women with nonsevere or severe stage 2 hypertension, as discrete diagnostic categories, experienced more adverse outcomes than women with normal blood pressure (risk ratios 1.29-5.88). Using blood pressure categories as diagnostic thresholds (women with blood pressure within the category or any higher category vs those with blood pressure in any lower category), dose-response relationships were observed between increasing thresholds and adverse outcomes, but likelihood ratios were informative only for severe stage 2 hypertension and maternal CNS events (likelihood ratio $6 \cdot 36$ [95\% CI 3.65-11.07]) and perinatal death $(5 \cdot 07$ [3.64-7.07]), particularly stillbirth (8.53 $[5 \cdot 63-12 \cdot 92])$.

Interpretation In low-resource settings, neither elevated blood pressure nor stage 1 hypertension were associated with maternal, fetal, or neonatal mortality or morbidity adverse composite outcomes. Only the threshold for severe stage 2 hypertension met diagnostic test performance standards. Current diagnostic thresholds for hypertension in pregnancy should be retained.

Funding University of British Columbia, the Bill \& Melinda Gates Foundation.

Copyright (C) 2021 The Author(s). Published by Elsevier Ltd. This is an Open Access article under the CC BY 4.0 license.

\section{Introduction}

Hypertension in pregnancy has traditionally been defined as a systolic blood pressure (sBP) of at least $140 \mathrm{~mm} \mathrm{Hg}$ or a diastolic blood pressure (dBP) of at least $90 \mathrm{~mm} \mathrm{Hg}$, or both. ${ }^{1}$ Hypertension defined in this way identifies pregnant women at increased risk of pre-eclampsia and other maternal and fetal or neonatal complications, including death, and these women are recommended to receive enhanced antenatal care and monitoring worldwide.

In 2017, the American College of Cardiology and American Heart Association recommended lowering

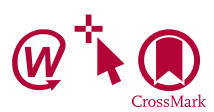

Lancet Glob Health 2021; 9: e1119-28

Published Online July 5, 2021 https://doi.org/10.1016/ S2214-109X(21)00219-9

See Comment page e1041

*Joint first authors

†Study group members are listed in the appendix (p 3)

Department of Obstetrics and Gynaecology and BC Children's Hospital Research Institute

(J N Bone MSc,

Prof LA Magee MD $M$ Vidler PhD,

Prof P von Dadelszen DPhil) and Centre for Health Evaluation and Outcome Sciences, Providence Health Care Research Institute

(Prof J Singer PhD), University of British Columbia,

Vancouver, BC, Canada Department of Women and Children's Health, School of Life Course Sciences, Faculty of Life Sciences and Medicine, King's College London, London, UK (Prof L A Magee, H Nathan PhD, Prof A Shennan MD, Prof $\mathrm{P}$ von Dadelszen); Centre of Excellence, Division of Woman and Child Health, Aga Khan University, Karachi, Pakistan (Prof R N Qureshi MBBS, Prof Z A Bhutta PhD); Centro de Investigação em Saúde de Manhiça, Manhiça, Mozambique (C Sacoor MSc, E Sevene PhD, K Munguambe PhD); Department of Physiological Sciences, Clinical Pharmacology, Faculdade de Medicina, Universidade Eduardo Mondlane, Maputo, Mozambique (E Sevene); KLE Academy of Higher Education and Research's J N Medical College, Belagavi, Karnataka, India (Prof M B Bellad MD, Prof S S Goudar MD); 


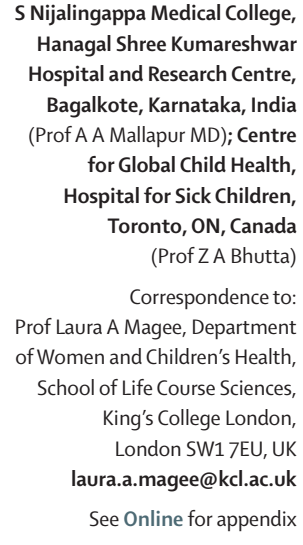

\section{Research in context}

\section{Evidence before this study}

We searched MEDLINE, PubMed, Embase, CINAHL, CNTRAL,

LILACS, Web of Science, and Google Scholar databases,

including reference lists of eligible studies, for studies published in English between Jan 1, 2017, and Dec 31, 2020, using the search terms "human" AND ("hypertension" OR "hypertensive disorders of pregnancy" OR "pregnancy-induced hypertension" OR "preeclampsia" OR "pregnancy toxemias" OR "gestational hypertension") AND ("American College of Cardiology" OR "American Heart Association") AND ("stage 1 hypertension" OR "prehypertension") AND ("Pregnancy[mh]" OR "Pregnan*" OR "Gestation*" OR "pregnant women[mh]" OR "Pregnancy Complications[mh]" OR "Postpartum Period"[Mesh] OR "Puerperium" OR "postpartum" OR "Peripartum Period"[Mesh] OR "Peripartum*" OR "Perinatal Care[mh]" OR "perinatal").

In 2017, the American College of Cardiology and American Heart Association recommended lowering blood pressure thresholds for diagnosing hypertension outside of pregnancy. Several studies have examined the risk of adverse pregnancy outcomes associated with these lower blood pressure values in pregnancy, compared with the established cutoff of $140 / 90 \mathrm{~mm} \mathrm{Hg}$ or greater.

\section{Added value of this study}

Nearly all evidence for using the American College of Cardiology and American Heart Association thresholds to diagnose hypertension in pregnancy are from high-income settings and rely on retrospective, routinely collected clinical data. Furthermore, these studies have focused exclusively on associative measures (such as risk ratios) between blood pressure thresholds and adverse outcomes. By contrast, this study provides prospective blood pressure data from more than 20000 pregnant women, in three low-income and middle-income countries (LMICs), using standardised measurement technique and a validated device, and examines not only associations between blood pressure thresholds and adverse outcomes, but also the diagnostic test properties of those thresholds. Associations were dependent on blood pressure greater than or equal to $160 / 110 \mathrm{~mm} \mathrm{Hg}$.

\section{Implications of all the available evidence}

Our findings suggest that there is an association between the American College of Cardiology and American Heart Association blood pressure thresholds and adverse pregnancy outcomes in LMIC settings, but there is no antenatal blood pressure threshold that is sensitive with regards to the adverse maternal, fetal, or neonatal outcomes studied, including datadriven cutoffs. However, severe stage 2 hypertension (blood pressure $\geq 160 / 110 \mathrm{~mm} \mathrm{Hg}$ ) is associated with a substantially increased risk of adverse maternal CNS outcomes and fetal or neonatal death, particularly stillbirth, and should be treated as per international guidance. Antenatal care must aim to provide more than accurate blood pressure measurement to achieve the Countdown 2030 goals. the blood pressure thresholds for diagnosing hypertension outside of pregnancy, classified as: elevated blood pressure (or elevated SBP; defined as SBP $120-129 \mathrm{~mm} \mathrm{Hg}$ and $\mathrm{dBP}<80 \mathrm{~mm} \mathrm{Hg}$ ), stage 1 hypertension (sBP $130-139 \mathrm{~mm} \mathrm{Hg}$ or dBP $80-89 \mathrm{~mm} \mathrm{Hg}$, or both), and stage 2 hypertension (sBP $\geq 140 \mathrm{~mm} \mathrm{Hg}$ or $\mathrm{dBP} \geq 90 \mathrm{~mm} \mathrm{Hg}$, or both). ${ }^{2}$ Although the American College of Obstetricians and Gynecologists and WHO have retained a definition of blood pressure greater than or equal to $140 / 90 \mathrm{~mm} \mathrm{Hg}$ for hypertension in pregnancy, several studies have reported a doseresponse relationship between increasing blood pressure and adverse pregnancy outcomes, across gestational ages; $;^{3,4}$ these findings provide potential support for the redefinition of hypertension in pregnancy. However, despite use of blood pressure as an essential screening test in pregnancy, no studies have yet reported the diagnostic test properties (such as sensitivity and specificity) of various blood pressure thresholds for hypertension diagnosis.

We aimed to analyse the relationship between blood pressure thresholds to define hypertension in pregnancy and adverse maternal, fetal, or neonatal outcomes, as well as the diagnostic test properties of these cutoffs, in low-resource settings.

\section{Methods}

\section{Study design and participants}

We did a secondary analysis of data from 22 intervention clusters in the Community-Level Interventions for Pre-eclampsia (CLIP) cluster randomised trials (NCT01911494) in India ( $n=6)$, Mozambique $(n=6)$, and Pakistan $(n=10) .^{5-8}$ The unit of randomisation (cluster) was the local administrative unit. We included pregnant women aged 15-49 years (12-49 years in Mozambique), identified in their community by trained community health workers, who had data on blood pressure measurements and outcomes. All women provided written informed consent to participate. The trial was unmasked given the nature of the intervention, aimed at addressing the so-called three delays in triage, transport, and treatment related to mortality risk, particularly associated with pre-eclampsia. ${ }^{9}$

Ethics approvals were granted by the University of British Columbia, Canada (H12-03497) and relevant incountry research ethics boards (Aga Khan University, Pakistan, 2590-Obs-ERC-13; KLE University, India, MDC/IECHSR/2011-12/A-4, ICMR 5/7/859/12-RHN; Centro de Investigação em Saúde de Manhiça, Mozambique, CIBS-CISM/038/14; and Mozambique National Bioethic Committee, 219/CNBS/14). 


\section{Procedures}

First, community engagement addressed barriers and facilitators to accessing care. Second, existing cadres of community health workers were trained to task-share pregnancy hypertension-oriented care at CLIP contacts in women's homes, using the CLIP Pre-eclampsia Integrated Estimate of Risk Score (PIERS) On-theMove (POM) digital health app for risk stratification. ${ }^{10}$ Community health workers responded to emergency conditions (if applicable); measured women's blood pressure and did dipstick urinalysis for proteinuria at the first and any subsequent contact where hypertension was identified; administered oral methyldopa $(750 \mathrm{mg}$ ) if blood pressure was greater than or equal to 160/110 $\mathrm{mm} \mathrm{Hg}$ and intramuscular magnesium sulphate $(10 \mathrm{~g})$ if severe pre-eclampsia (defined as at least one of: $\mathrm{sBP} \geq 160 \mathrm{~mm} \mathrm{Hg}$, mini pre-eclampsia integrated estimate of risk [miniPIERS] probability $\geq 25 \%$, eclampsia, or stroke) was suspected; and referred women to a comprehensive emergency obstetric care facility if advised by POM. Antenatal POM-guided visits (including blood pressure measurement) were scheduled monthly from enrolment.

Blood pressure measurement for all women in the intervention clusters (and, therefore, in this analysis) was standardised and taken by trained community health workers, using a semi-automated pregnancyvalidated and pre-eclampsia-validated oscillometric device (Microlife 3AS1-2; Microlife, Taipei, Taiwan).$^{11}$ Women were instructed to rest for $5 \mathrm{~min}$, then their blood pressure was measured at least twice, with a third measurement taken if the first two readings differed by $10 \mathrm{mmHg}$ or more. All readings were entered into the POM app, with blood pressure for the visit calculated as the mean of the first two readings, or of the second and third if three readings were taken. All readings were stored electronically in REDCap databases.

Trained surveillance teams did regular household surveys (every 3-6 months) in Mozambique and Pakistan; in India, a prospective population-based surveillance system was established. The PRE-eclampsia Eclampsia Monitoring, Prevention, and Treatment (PRE-EMPT) research group, University of British Columbia, Canada, was responsible for overall trial coordination and data management.

\section{Outcomes}

The primary CLIP composite outcome was a composite of maternal, fetal, and neonatal mortality and morbidity, and all outcomes were adjudicated by an in-country team of clinicians. Maternal death and morbidity were assessed during pregnancy or within 42 days after pregnancy; morbidity was defined as one or more lifethreatening pregnancy complications (a serious endorgan complication of pre-eclampsia [ie, eclampsia, stroke, coma, antepartum haemorrhage, or disseminated intravascular coagulation], another major maternal complication [ie, obstetric sepsis, or vesicovaginal or rectovaginal fistula], or receipt of a life-saving intervention [ie, cardiopulmonary resuscitation, mechanical ventilation, blood transfusion, interventions for major post-partum haemorrhage, or dialysis]). This analysis also included a maternal CNS composite outcome of one or more of maternal eclampsia, stroke, coma, or mortality. Fetal or neonatal death included stillbirth,

\begin{tabular}{|c|c|}
\hline & Participants $(n=21069)$ \\
\hline \multicolumn{2}{|l|}{ Country } \\
\hline India & $6067(28 \cdot 8 \%)$ \\
\hline Mozambique & $4163(19 \cdot 8 \%)$ \\
\hline Pakistan & $10839(51 \cdot 4 \%)$ \\
\hline Maternal age, years & $25 \cdot 0(22 \cdot 0-30 \cdot 0)$ \\
\hline Maternal basic level of education* & 8409 (39.9\%) \\
\hline Gestational age at enrolment, weeks & $17 \cdot 1(11 \cdot 2-24 \cdot 1)$ \\
\hline Enrolment at $\geq 20$ weeks of gestation & $7832(37 \cdot 2 \%)$ \\
\hline Parous & $15099(71 \cdot 7 \%)$ \\
\hline \multicolumn{2}{|l|}{ Blood pressure, $\mathrm{mm} \mathrm{Hg}$} \\
\hline Mean sBP & $106 \cdot 0(100 \cdot 4-112 \cdot 0)$ \\
\hline Mean dBP & $66 \cdot 6(62 \cdot 5-71 \cdot 0)$ \\
\hline Maximum sBP & $113 \cdot 0(106 \cdot 0-120 \cdot 0)$ \\
\hline Maximum dBP & $73 \cdot 0(67 \cdot 0-79 \cdot 0)$ \\
\hline \multicolumn{2}{|l|}{ Maximal blood pressure category $\dagger$} \\
\hline Normal blood pressure & $13780(65 \cdot 4 \%)$ \\
\hline Elevated blood pressure & $2196(10 \cdot 4 \%)$ \\
\hline Stage 1 hypertension & $3751(17 \cdot 8 \%)$ \\
\hline Non-severe stage 2 hypertension & $1178(5 \cdot 6 \%)$ \\
\hline Severe stage 2 hypertension & $164(0.8 \%)$ \\
\hline Gestational age at delivery, weeks & $39 \cdot 0(37 \cdot 0-40 \cdot 4)$ \\
\hline Preterm delivery ( $<37$ weeks of gestation) & $4651(22 \cdot 1 \%)$ \\
\hline \multicolumn{2}{|l|}{ Outcomes } \\
\hline Primary CLIP composite & $4816(22 \cdot 9 \%)$ \\
\hline Maternal mortality & $43(0 \cdot 2 \%)$ \\
\hline Maternal morbidity & $2006(9 \cdot 5 \%)$ \\
\hline Maternal CNS composite & $282(1 \cdot 3 \%)$ \\
\hline Fetal or neonatal death & $1657(7 \cdot 9 \%)$ \\
\hline Stillbirth & $810(3 \cdot 8 \%)$ \\
\hline
\end{tabular}

Data are $\mathrm{n}(\%)$ or median (IQR) unless otherwise stated. sBP=systolic blood pressure. $\mathrm{dBP}=$ diastolic blood pressure. $\mathrm{CLIP}=\mathrm{Community}$-Level Interventions for Pre-eclampsia. *Basic level of education was defined as at least 8 years of schooling in India, at least attainment of grade 5 in Mozambique, or at least 5 years of schooling in Pakistan. †Blood pressure was categorised as: normal blood pressure ( $\mathrm{sBP}<120 \mathrm{~mm} \mathrm{Hg}$ and $\mathrm{dBP}<80 \mathrm{~mm} \mathrm{Hg}$ ), elevated blood pressure (sBP $120-129 \mathrm{~mm} \mathrm{Hg}$ and $\mathrm{dBP}<80 \mathrm{~mm} \mathrm{Hg}$ ), stage 1 hypertension (sBP 130-139 mm Hg or dBP 80-89 mm Hg, or both), non-severe stage 2 hypertension (sBP 140-159 mm Hg or dBP 90-109 mm Hg, or both), or severe stage 2 hypertension ( $\mathrm{sBP} \geq 160 \mathrm{~mm} \mathrm{Hg}$ or $\mathrm{dBP} \geq 110 \mathrm{~mm} \mathrm{Hg}$, or both). $\ddagger$ The primary CLIP composite outcome was a composite of maternal and perinata mortality and morbidity. Maternal mortality or morbidity were assessed during pregnancy or within 42 days after pregnancy; morbidity was defined as one or more life-threatening pregnancy complications (ie, a serious end-organ complication of pre-eclampsia, another major cause of maternal mortality, or receipt of a life-saving intervention). The maternal CNS composite outcome was one or more of maternal eclampsia, stroke, coma, or mortality. Fetal or neonatal death included stillbirth and early or late neonatal mortality (appendix p 4).

Table 1: Baseline characteristics 


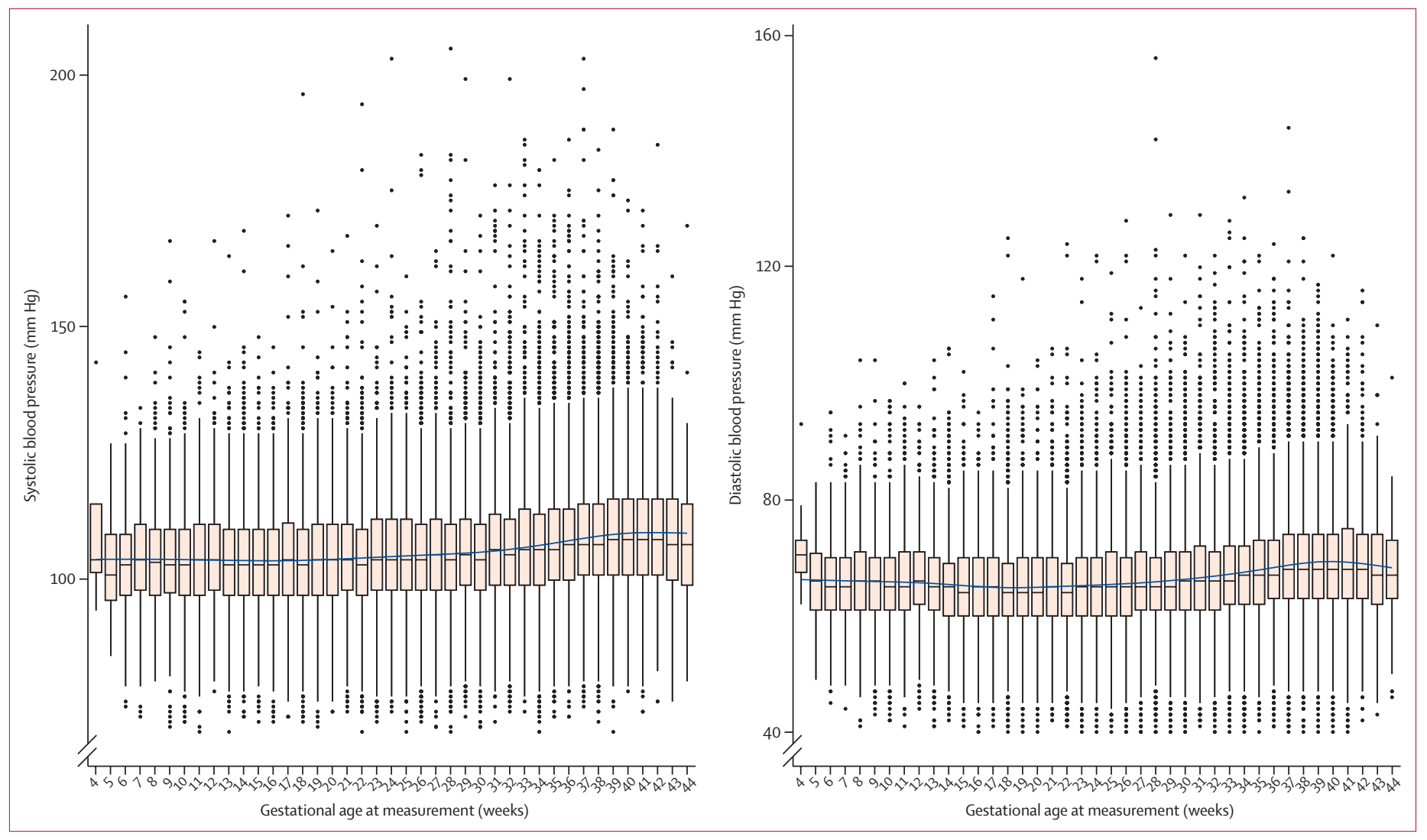

Figure 1: Maximal blood pressure measurements by gestational age

Maximal blood pressure values per woman are shown, as a median with IQR for each gestational age week.

early neonatal mortality, or late neonatal mortality (appendix p 4).

In this analysis, we included CLIP trial participants who were from intervention groups and had at least one POM app-guided antenatal contact with blood pressure measurement done by community health workers at a clinically estimated gestation of 44 weeks or less. Analyses including post-partum and perinatal outcomes were restricted to women who had delivered and provided outcome information.

We classified the antenatal blood pressure readings for each woman at each visit, on the basis of the American College of Cardiology and American Heart Association criteria, ${ }^{2}$ as: normal blood pressure $(\mathrm{sBP}<120 \mathrm{~mm} \mathrm{Hg}$ and $\mathrm{dBP}<80 \mathrm{~mm} \mathrm{Hg}$ ), elevated blood pressure (sBP $120-129 \mathrm{~mm} \mathrm{Hg}$ and $\mathrm{dBP}<80 \mathrm{~mm} \mathrm{Hg}$ ), stage 1 hypertension (sBP 130-139 mm Hg or dBP 80-89 mm Hg, or both), non-severe stage 2 hypertension (sBP 140-159 mm Hg or dBP 90-109 mm Hg, or both), and severe stage 2 hypertension ( $\mathrm{sBP} \geq 160 \mathrm{~mm} \mathrm{Hg}$ or $\mathrm{dBP}$ $\geq 110 \mathrm{~mm} \mathrm{Hg}$, or both). We classified women according to the maximum blood pressure category reached across all visits for the primary analyses. Women who were hypertensive before 20 weeks of gestation were regarded as having chronic hypertension, and those who were hypertensive at 20 weeks of gestation or greater were regarded as having gestational hypertension or preeclampsia. $^{12}$

\section{Statistical analysis}

Descriptive statistics were used to summarise baseline maternal characteristics, and maximal blood pressure categories overall and according to gestational age at measurement ( $<20$ weeks $v s \geq 20$ weeks).

The possible dose-response relationship between blood pressure category and adverse outcomes was assessed in two ways. First, we treated each category as mutually exclusive and calculated the risk ratio (RR) between normal blood pressure and each category, using generalised estimating equations with a Poisson link function. ${ }^{13}$ Second, we fit analogous models, but treated the lower limit of each category as a blood pressure cutoff for diagnosis of hypertension. For example, for stage 1 hypertension, we compared women with sBP $130 \mathrm{~mm} \mathrm{Hg}$ or greater or dBP $80 \mathrm{~mm} \mathrm{Hg}$ or greater (or both), with those who had sBP less than $130 \mathrm{~mm} \mathrm{Hg}$ and dBP less than $80 \mathrm{~mm} \mathrm{Hg}$. All models were adjusted for maternal age, maternal basic level of education (ie, $\geq 8$ years of schooling in India, attainment of at least grade 5 in Mozambique, or $\geq 5$ years of 
schooling in Pakistan), gestational age at enrolment, and nulliparity. SEs were based on the sandwich estimator to account for clustering.

The diagnostic test properties of blood pressure categories were assessed using sensitivity, specificity, and positive and negative likelihood ratios (LRs). Positive LR was calculated as: sensitivity / (1-specificity). Negative LR was calculated as: (1- sensitivity) / specificity. CIs were calculated by standard methods. ${ }^{14}$ For each calculation, women with blood pressure equal to or higher than the given blood pressure threshold were compared with those with blood pressure lower than the threshold. LRs describe the likelihood that a given test result would alter the probability of a diagnosis; positive LR values were interpreted as good if greater than or equal to $5 \cdot 0$ and negative LR values were interpreted as good if less than $0 \cdot 2$.

In a sensitivity analysis to determine if the sensitivity of blood pressure categories for adverse outcomes improved closer to term, we re-classified women according to the maximum blood pressure category reached within predefined gestational age categories ( 28 weeks to $<32$ weeks, 32 weeks to $<37$ weeks, and $\geq 37$ weeks of gestation). Also, we estimated the optimal sBP and dBP cutoffs to maximise sensitivity of blood pressure for fixed false-positive rates of $5 \%, 10 \%$, and $20 \%$. All analyses were done using $\mathrm{R}$ version 4.0.2. ${ }^{15}$

\section{Role of the funding source}

The funder of the study had no role in study design, data collection, data analysis, data interpretation, or writing of the report.

\section{Results}

Between Nov 1, 2014, and Feb 28, 2017, 21069 women (6067 in India, 4163 in Mozambique, and 10839 in Pakistan) contributed 103679 blood pressure measurements across the three CLIP trials (table 1). Most women were aged in their mid-20s, had less than a basic level of education, and were parous. In general, women were enrolled in CLIP late in the first trimester or early in the second trimester, with most enrolling at less than 20 weeks of gestation. About two-thirds of women had normal blood pressure throughout pregnancy. Slightly more than one-quarter of women had abnormal blood pressure classified as elevated blood pressure (2196 [10.4\%] of 21069) or non-severe stage 1 hypertension (3751 [17.8\%]). Stage 2 hypertension (non-severe or severe) occurred in 1342 (6.4\%) of 21069 women. Most women delivered at term, but one-quarter delivered preterm. Another quarter suffered a maternal, fetal, or neonatal complication, which were mostly morbidity for the mother (about one in ten women) or death of the fetus (42 per 1000 livebirths) or neonate (41 per 1000 livebirths). Blood pressure was only 1-2 $\mathrm{mmHg}$ higher among women with adverse outcomes than in women without adverse outcomes (appendix p 5).

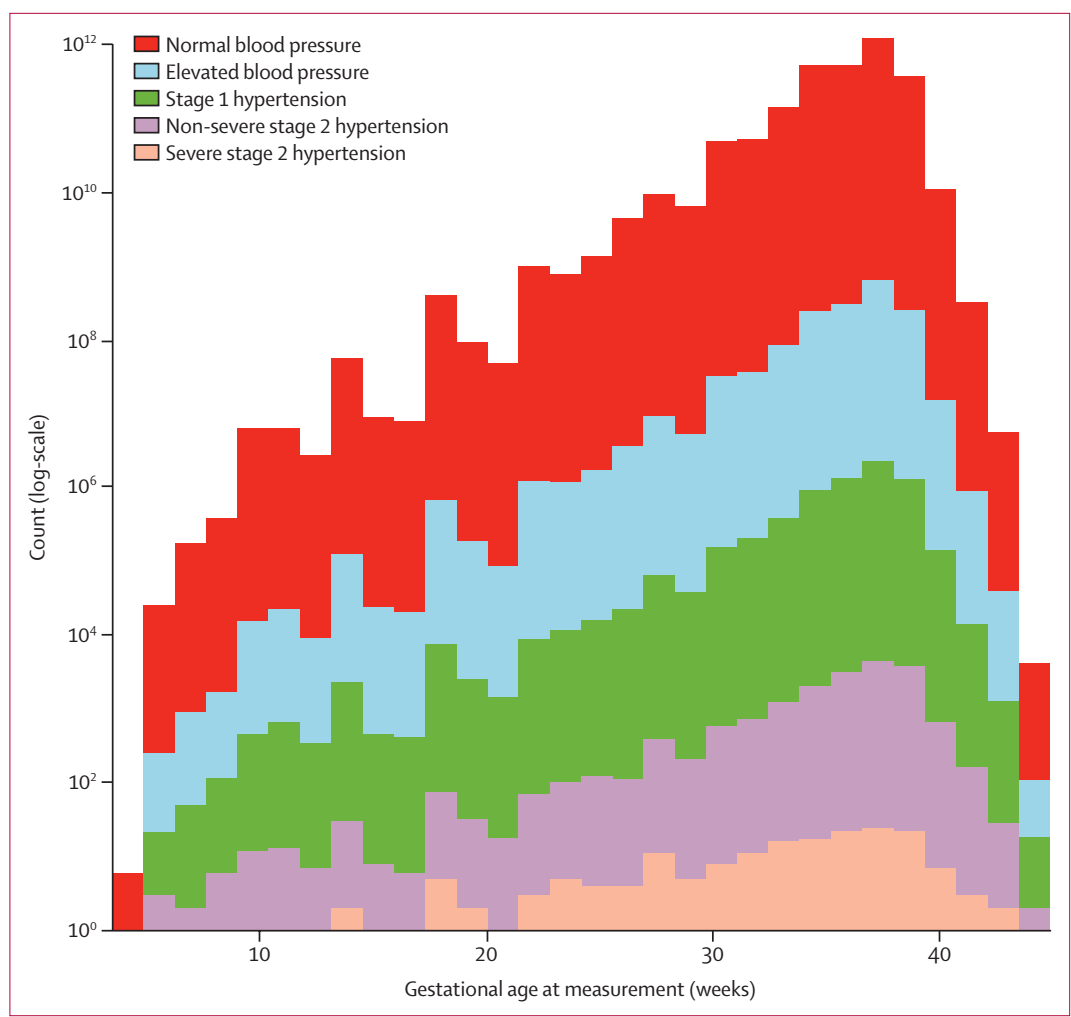

Figure 2: Maximal blood pressure measurements as blood pressure categories by gestational age A log-scale is used for the $y$-axis (number of women) for clarity. Blood pressure was categorised as: normal blood pressure (sBP $<120 \mathrm{~mm} \mathrm{Hg}$ and $\mathrm{dBP}<80 \mathrm{~mm} \mathrm{Hg}$ ), elevated blood pressure (sBP $120-129 \mathrm{~mm} \mathrm{Hg}$ and $\mathrm{dBP}<80 \mathrm{~mm} \mathrm{Hg}$ ), stage 1 hypertension (sBP 130-139 mm Hg or dBP 80-89 mm Hg, or both), non-severe stage 2 hypertension (sBP 140-159 mm Hg or dBP 90-109 mm Hg, or both), or severe stage 2 hypertension ( $\mathrm{sBP} \geq 160 \mathrm{~mm} \mathrm{Hg}$ or $\mathrm{dBP} \geq 110 \mathrm{~mm} \mathrm{Hg}$, or both).

Blood pressure values overall were stable, without a clinically important mid-trimester decrease, until about 30 weeks of gestation, after which both SBP and dBP increased with advancing gestational age (figure 1). Maximal blood pressure values classified as elevated blood pressure, stage 1 hypertension, and stage 2 hypertension (non-severe or severe) were more common with advancing gestational age (figure 2). Blood pressure in most women remained at the same category or decreased after 20 weeks of gestation or longer, regardless of whether blood pressure at less than 20 weeks of gestation was classified as normal blood pressure (5160 [63.0\%] of 8194 women), elevated blood pressure $(336[60.8 \%]$ of 552$)$, stage 1 hypertension (527 [75.7\%] of 696), or non-severe stage 2 hypertension (91 [75.8\%] of 120 ; table 2 ). If the stage 1 hypertension category cutoffs were used as the new threshold for diagnosing hypertension in pregnancy, an additional $1681(17 \cdot 5 \%)$ of 9574 women would be diagnosed; if elevated blood pressure category cutoffs were used, an additional 1021 (10.7\%) of women would be diagnosed (table 2).

There was a dose-response relationship between higher blood pressure category and greater RR for 


\begin{tabular}{|c|c|c|c|c|c|c|}
\hline & $\begin{array}{l}\text { Normal blood } \\
\text { pressure at } \\
\geq 20 \text { weeks } \\
(n=13346)\end{array}$ & $\begin{array}{l}\text { Elevated blood } \\
\text { pressure at } \\
\geq 20 \text { weeks } \\
(n=2024)\end{array}$ & $\begin{array}{l}\text { Stage } 1 \\
\text { hypertension } \\
\text { at } \geq 20 \text { weeks } \\
(n=3420)\end{array}$ & $\begin{array}{l}\text { Non-severe } \\
\text { stage } 2 \\
\text { hypertension } \\
\text { at } \geq 20 \text { weeks } \\
(\mathrm{n}=1095)\end{array}$ & $\begin{array}{l}\text { Severe stage } 2 \\
\text { hypertension } \\
\text { at } \geq 20 \text { weeks } \\
(n=154)\end{array}$ & $\begin{array}{l}\text { No blood } \\
\text { pressure } \\
\text { values at } \\
\geq 20 \text { weeks } \\
(\mathrm{n}=1030)\end{array}$ \\
\hline Normal blood pressure at <20 weeks $(n=8194)$ & 5160 & $685^{*}$ & $1035^{*} \dagger$ & 378 & 48 & 888 \\
\hline Elevated blood pressure at $<20$ weeks $(n=552$ ) & $219^{*}$ & $117^{*}$ & $119^{*} \dagger$ & 49 & 0 & 48 \\
\hline Stage 1 hypertension at <20 weeks ( $n=696$ ) & $206^{*} \dagger$ & $87^{*} \dagger$ & $234^{*} \dagger$ & 83 & 11 & 75 \\
\hline Non-severe stage 2 hypertension at <20 weeks $(n=120)$ & 27 & 7 & 36 & 21 & 13 & 16 \\
\hline Severe stage 2 hypertension at $<20$ weeks $(n=12)$ & 2 & 1 & 1 & 3 & 2 & 3 \\
\hline No blood pressure values at $<20$ weeks ( $n=11495$ ) & 7732 & 1127 & 1995 & 561 & 80 & 0 \\
\hline \multicolumn{7}{|c|}{$\begin{array}{l}\text { Data are } n \text {. Blood pressure was categorised as: normal blood pressure (sBP }<120 \mathrm{~mm} \mathrm{Hg} \text { and dBP }<80 \mathrm{~mm} \mathrm{Hg} \text { ), elevated blood pressure (sBP } 120-129 \mathrm{~mm} \mathrm{Hg} \text { and } \\
\mathrm{dBP}<80 \mathrm{~mm} \mathrm{Hg} \text { ), stage } 1 \text { hypertension ( } \mathrm{sBP} 130-139 \mathrm{~mm} \mathrm{Hg} \text { or dBP } 80-89 \mathrm{~mm} \mathrm{Hg} \text {, or both), non-severe stage } 2 \text { hypertension (sBP } 140-159 \mathrm{~mm} \mathrm{Hg} \text { or dBP } 90-109 \mathrm{~mm} \mathrm{Hg} \text {, } \\
\text { or both), or severe stage } 2 \text { hypertension ( }(\mathrm{BPP} \geq 160 \mathrm{~mm} \mathrm{Hg} \text { or dBP } \geq 110 \mathrm{~mm} \mathrm{Hg} \text {, or both). sBP=systolic blood pressure. dBP=diastolic blood pressure. * Indicates women who } \\
\text { would additionally be diagnosed with hypertension if elevated blood pressure was used as the threshold for diagnosing hypertension in pregnancy. +Indicates women } \\
\text { who would additionally be diagnosed with hypertension if stage } 1 \text { hypertension was used as the threshold for diagnosing hypertension in pregnancy. }\end{array}$} \\
\hline
\end{tabular}

\begin{tabular}{|c|c|c|c|c|c|}
\hline & $\begin{array}{l}\text { Normal blood } \\
\text { pressure }\end{array}$ & $\begin{array}{l}\text { Elevated blood } \\
\text { pressure }\end{array}$ & Stage 1 hypertension & $\begin{array}{l}\text { Non-severe stage } 2 \\
\text { hypertension }\end{array}$ & $\begin{array}{l}\text { Severe stage } 2 \\
\text { hypertension }\end{array}$ \\
\hline \multicolumn{6}{|c|}{ Comparison with normal blood pressure category } \\
\hline CLIP composite & 1 (ref) & $0.99(0.94-1.03)$ & $1.08(1.00-1.16)^{*} \dagger$ & $1.29(1.13-1.47) \dagger$ & $2 \cdot 48(2 \cdot 06-2 \cdot 98) \dagger$ \\
\hline Maternal composite & 1 (ref) & $1.02(0.89-1.17)$ & $1.11(0.93-1 \cdot 33)$ & $1.20(0.90-1.59)$ & $2 \cdot 40(1.60-3.59) \dagger$ \\
\hline Maternal CNS composite & 1 (ref) & $0.98(0.63-1.51)$ & $1.26(0.91-1 \cdot 75)$ & $1.46(1.00-2.13) \dagger$ & $6.05(3.88-9.46) \dagger$ \\
\hline Fetal or neonatal death & 1 (ref) & $0.93(0.79-1.09)$ & $1.15(0.96-1 \cdot 38)$ & $1.48(1.10-1.97) \dagger$ & $4.09(3.02-5.55) \dagger$ \\
\hline Stillbirth & 1 (ref) & $0.98(0.74-1.29)$ & $1.24(0.97-1.58)$ & $1 \cdot 81(1 \cdot 30-2 \cdot 54) \dagger$ & $5.88(3.95-8.73) \dagger$ \\
\hline \multicolumn{6}{|c|}{ Comparison of blood pressure category and all higher categories, with all lower blood pressure categoriesł } \\
\hline CLIP composite &.. & $1.12(1.05-1.19) \dagger$ & $1 \cdot 17(1 \cdot 09-1 \cdot 26) \dagger$ & $1.41(1 \cdot 24-1 \cdot 60) \dagger$ & $2 \cdot 48(2 \cdot 06-2 \cdot 98) \dagger$ \\
\hline Maternal composite &.. & $1.13(0.96-1.33)$ & $1.17(0.98-1.40)$ & $1.32(1.05-1.65) \dagger$ & $2.40(1.60-3.59) \dagger$ \\
\hline Maternal CNS composite &.. & $1.32(1.08-1.61) \dagger$ & $1.47(1.13-1.90) \dagger$ & $1.91(1.41-2.57) \dagger$ & $6.05(3.88-9.46) \dagger$ \\
\hline Fetal or neonatal death & $\cdot$. & $1.20(1.02-1.43) \dagger$ & $1.33(1.12-1.58) \dagger$ & $1 \cdot 75(1 \cdot 42-2 \cdot 15) \dagger$ & $4.09(3 \cdot 02-5 \cdot 55) \dagger$ \\
\hline Stillbirth & .. & $1.36(1.09-1 \cdot 70) \dagger$ & $1.52(1.24-1.87) \dagger$ & $2 \cdot 19(1 \cdot 72-2 \cdot 79) \dagger$ & $5.88(3.95-8 \cdot 73) \dagger$ \\
\hline \multicolumn{6}{|c|}{ 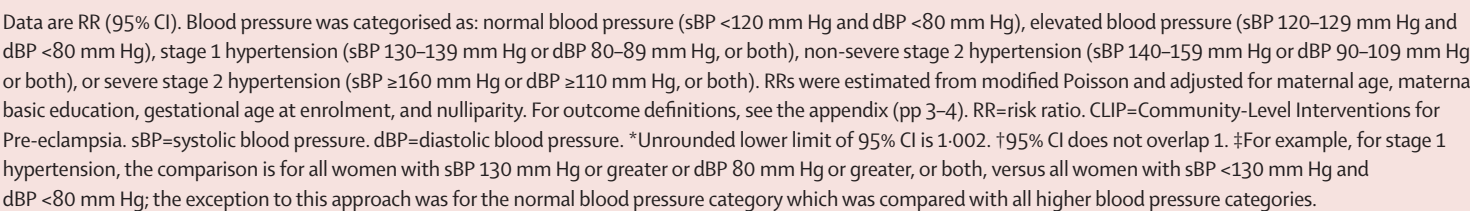 } \\
\hline
\end{tabular}

adverse outcomes compared with normal blood pressure, for most outcomes with non-severe stage 2 hypertension and for all outcomes with severe stage 2 hypertension, which increased risk by at least two times and up to six times (table 3). When the diagnostic criteria for each blood pressure category were used as a threshold for diagnosis of an abnormal blood pressure, the risk of all adverse outcomes, other than the maternal composite, increased from elevated blood pressure onwards; however, the point estimates for elevated blood pressure, stage 1 hypertension, and non-severe stage 2 hypertension were consistently higher than they were when these blood pressure categories were each compared with normal blood pressure, and RRs were less than $2 \cdot 00$ for all but non-severe stage 2 hypertension and stillbirth.

The only blood pressure category that was useful as a diagnostic test for the outcomes examined was severe stage 2 hypertension; sensitivity was low, but the positive LR was good for the maternal CNS composite outcome and fetal or neonatal death, particularly stillbirth (table 4). All other blood pressure categories, including non-severe stage 2 hypertension, had low sensitivity $(<45 \%)$ for all outcomes, and uninformative positive LR and negative LR values.

In sensitivity analyses restricted to blood pressure measurements in specific gestational age ranges within the third trimester, sensitivity remained low for all 


\begin{tabular}{|c|c|c|c|c|c|}
\hline & Events, $\mathrm{n}(\%)^{*}$ & Sensitivity $(95 \% \mathrm{Cl})$ & Specificity $(95 \% \mathrm{Cl})$ & Positive LR $(95 \% \mathrm{Cl}) \dagger$ & Negative LR $(95 \% \mathrm{Cl}) \ddagger$ \\
\hline \multicolumn{6}{|l|}{ CLIP composite } \\
\hline Normal blood pressure ( $n=13441)$ & $2987(22 \cdot 2 \%)$ & & & & .. \\
\hline Elevated blood pressure $(n=2149)$ & $484(22 \cdot 5 \%)$ & $38.0 \%(36 \cdot 6-39 \cdot 4)$ & $66 \cdot 3 \%(65 \cdot 5-67 \cdot 0)$ & $1.13(1.08-1.17)$ & $0.94(0.91-0.96)$ \\
\hline Stage 1 hypertension $(\mathrm{n}=3679)$ & $937(25 \cdot 5 \%)$ & $27 \cdot 9 \%(26 \cdot 7-29 \cdot 2)$ & $76 \cdot 8 \%(76 \cdot 1-77 \cdot 5)$ & $1.20(1.14-1.27)$ & $0.94(0.92-0.96)$ \\
\hline Non-severe stage 2 hypertension ( $\mathrm{n}=1163$ ) & $321(27 \cdot 6 \%)$ & $8.5 \%(7 \cdot 7-9 \cdot 0)$ & $94 \cdot 2 \%(93 \cdot 8-94 \cdot 6)$ & $1.46(1.30-1.63)$ & $0.97(0.96-0.98)$ \\
\hline Severe stage 2 hypertension ( $n=161$ ) & $87(54 \cdot 0 \%)$ & $1 \cdot 8 \%(1 \cdot 4-2 \cdot 2)$ & $99 \cdot 5 \%(99 \cdot 4-99 \cdot 6)$ & $3.85(2 \cdot 83-5 \cdot 24)$ & $0.99(0.98-0.99)$ \\
\hline \multicolumn{6}{|l|}{ Maternal composite } \\
\hline Normal blood pressure $(n=13424)$ & $1253(9 \cdot 3 \%)$ & .. & .. & .. & .. \\
\hline Elevated blood pressure $(n=2146)$ & $212(9 \cdot 9 \%)$ & $38 \cdot 2 \%(36 \cdot 1-40 \cdot 4)$ & $65 \cdot 7 \%(65 \cdot 0-66 \cdot 3)$ & $1.11(1.05-1.18)$ & $0.94(0.91-0.97)$ \\
\hline Stage 1 hypertension $(\mathrm{n}=3674)$ & $409(11 \cdot 1 \%)$ & $27 \cdot 8 \%(25 \cdot 9-29 \cdot 8)$ & $76 \cdot 1 \%(75 \cdot 5-76 \cdot 7)$ & $1.16(1.08-1.25)$ & $0.95(0.92-0.98)$ \\
\hline Non-severe stage 2 hypertension ( $\mathrm{n}=1163$ ) & $120(10 \cdot 3 \%)$ & $7 \cdot 6 \%(6 \cdot 5-8 \cdot 9)$ & $93 \cdot 7 \%(93 \cdot 3-94 \cdot 0)$ & $1.21(1.03-1.42)$ & $0.99(0.97-1.00)$ \\
\hline Severe stage 2 hypertension $(\mathrm{n}=160)$ & $35(21 \cdot 9 \%)$ & $1 \cdot 7 \%(1 \cdot 2-2 \cdot 4)$ & $99 \cdot 3 \%(99 \cdot 2-99 \cdot 4)$ & $2.56(1.76-3.71)$ & $0.99(0.98-1.00)$ \\
\hline \multicolumn{6}{|l|}{ Maternal CNS composite } \\
\hline Normal blood pressure $(n=13413)$ & $161(1 \cdot 2 \%)$ & .. & .. & .. & .. \\
\hline Elevated blood pressure $(\mathrm{n}=2145)$ & $29(1 \cdot 4 \%)$ & $42 \cdot 9 \%(37 \cdot 1-48 \cdot 9)$ & $65 \cdot 4 \%(64 \cdot 7-66 \cdot 0)$ & $1.24(1.08-1.42)$ & $0.87(0.79-0.97)$ \\
\hline Stage 1 hypertension $(\mathrm{n}=3671)$ & $56(1.5 \%)$ & $32 \cdot 6 \%(27 \cdot 2-38 \cdot 4)$ & $75 \cdot 8 \%(75 \cdot 2-76 \cdot 4)$ & $1 \cdot 35(1.14-1 \cdot 60)$ & $0.89(0.82-0.96)$ \\
\hline Non-severe stage 2 hypertension ( $\mathrm{n}=1163$ ) & $23(2 \cdot 0 \%)$ & $12 \cdot 8 \%(9 \cdot 1-17 \cdot 2)$ & $93 \cdot 7 \%(93 \cdot 3-94 \cdot 0)$ & $2.01(1.48-2 \cdot 74)$ & $0.93(0.89-0.97)$ \\
\hline Severe stage 2 hypertension $(\mathrm{n}=160)$ & $13(8 \cdot 1 \%)$ & $4 \cdot 6 \%(2 \cdot 5-7 \cdot 8)$ & $99 \cdot 3 \%(99 \cdot 1-99 \cdot 4)$ & $6.36(3.65-11.07)$ & $0.96(0.94-0.99)$ \\
\hline \multicolumn{6}{|l|}{ Fetal or neonatal death } \\
\hline Normal blood pressure ( $\mathrm{n}=12$ 902) & $985(7 \cdot 6 \%)$ & . & .. & & .. \\
\hline Elevated blood pressure $(\mathrm{n}=2126)$ & $155(7 \cdot 3 \%)$ & $40 \cdot 6 \%(38 \cdot 2-43 \cdot 0)$ & $65 \cdot 0 \%(64 \cdot 3-65 \cdot 7)$ & $1 \cdot 16(1 \cdot 09-1 \cdot 23)$ & $0.91(0.88-0.95)$ \\
\hline Stage 1 hypertension $(\mathrm{n}=3647)$ & $337(9 \cdot 2 \%)$ & $31 \cdot 2 \%(29 \cdot 0-33 \cdot 5)$ & $75 \cdot 8 \%(75 \cdot 1-76 \cdot 4)$ & $1.29(1.19-1.39)$ & $0.91(0.88-0.94)$ \\
\hline Non-severe stage 2 hypertension ( $\mathrm{n}=1155$ ) & $130(11 \cdot 3 \%)$ & $10 \cdot 9 \%(9 \cdot 4-12 \cdot 5)$ & $93 \cdot 8 \%(93 \cdot 5-94 \cdot 2)$ & $1.76(1.51-2.04)$ & $0.95(0.93-0.97)$ \\
\hline Severe stage 2 hypertension $(\mathrm{n}=159)$ & $50(31 \cdot 4 \%)$ & $3 \cdot 0 \%(2 \cdot 2-4 \cdot 0)$ & $99 \cdot 4 \%(99 \cdot 3-99 \cdot 5)$ & $5.07(3.64-7.07)$ & $0.98(0.97-0.98)$ \\
\hline \multicolumn{6}{|l|}{ Stillbirth } \\
\hline Normal blood pressure ( $\mathrm{n}=12468)$ & $457(3.7 \%)$ & .. & .. & .. & .. \\
\hline Elevated blood pressure $(\mathrm{n}=2066)$ & $77(3 \cdot 7 \%)$ & $43 \cdot 6 \%(40 \cdot 1-47 \cdot 1)$ & $65 \cdot 0 \%(64 \cdot 3-65 \cdot 7)$ & $1 \cdot 24(1 \cdot 15-1 \cdot 35)$ & $0.87(0.82-0.92)$ \\
\hline Stage 1 hypertension $(\mathrm{n}=3496)$ & $167(4 \cdot 8 \%)$ & $34 \cdot 1 \%(30 \cdot 8-37 \cdot 5)$ & $76 \cdot 2 \%(75 \cdot 4-76 \cdot 9)$ & $1 \cdot 43(1 \cdot 29-1 \cdot 58)$ & $0.87(0.82-0.91)$ \\
\hline Non-severe stage 2 hypertension ( $\mathrm{n}=1112$ ) & $76(6 \cdot 8 \%)$ & $13 \cdot 5 \%(11 \cdot 2-16 \cdot 0)$ & $94 \cdot 8 \%(94 \cdot 4-95 \cdot 2)$ & $2 \cdot 59(2 \cdot 14-3 \cdot 13)$ & $0.91(0.89-0.94)$ \\
\hline Severe stage 2 hypertension ( $\mathrm{n}=151)$ & $33(21 \cdot 9 \%)$ & $4 \cdot 1 \%(2 \cdot 8-5 \cdot 7)$ & $99 \cdot 5 \%(99 \cdot 4-99 \cdot 6)$ & $8.53(5.63-12.92)$ & $0.96(0.95-0.98)$ \\
\hline \multicolumn{6}{|c|}{ 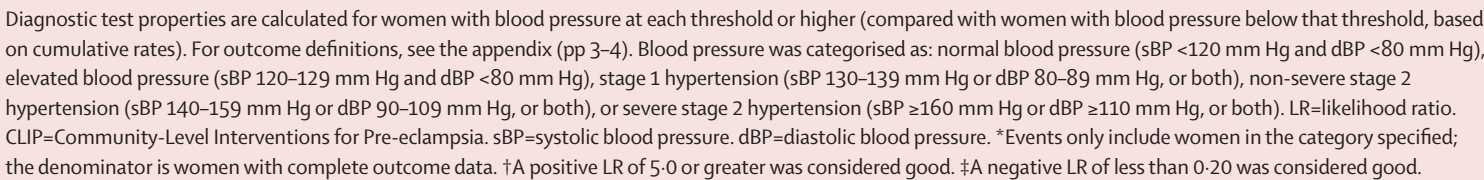 } \\
\hline
\end{tabular}

blood pressure categories, including the elevated blood pressure category, although sensitivity was slightly higher for measurements from 32 weeks to less than 37 weeks (appendix p 8). Also, sensitivity remained low $(<20 \%)$ for optimal sBP (123-136 mm $\mathrm{Hg})$ and dBP (81-104 $\mathrm{mm} \mathrm{Hg}$ ) cutoffs, for fixed false-positive rates of $5 \%, 10 \%$, and $20 \%$ (appendix p 6).

\section{Discussion}

In the CLIP trial clusters in low-resource settings, women classified as having elevated blood pressure or stage 1 hypertension did not have an increased risk of adverse maternal, fetal, or neonatal outcomes compared with women with normal blood pressure (<120/80 mm Hg). Risk of adverse outcomes was increased with stage 2 hypertension, particularly with severe stage 2 hypertension, which was associated with a two to six times increased risk compared with normal blood pressure. When blood pressure categories higher than normal were used as diagnostic cutoffs for abnormal blood pressure (how 140/90 mm Hg is currently used), the risk of adverse outcomes was increased; however, this increase was driven by the risks associated with severe stage 2 hypertension. In addition, sensitivities for adverse outcomes were low $(<45 \%)$, and no category other than severe stage 2 hypertension showed useful diagnostic test properties. Using optimal cutpoint analyses for falsepositive rates considered clinically reasonable led to poor sensitivity $(<30 \%)$ for all outcomes.

If elevated blood pressure was used as a diagnostic cutoff, an additional $28 \%$ of women would be labelled as having an abnormal blood pressure; if stage 1 
hypertension was used, this would be an additional $11 \%$ of women, in addition to women already identified as having hypertension by current criteria (ie, stage 2 hypertension in this study). In our study population, the prevalence of hypertension was previously found to be $14.0 \%$ in India, $16.8 \%$ in Mozambique, and $11.6 \%$ in Pakistan, after inclusion of hypertensive diagnoses from household survey and facility records. ${ }^{16}$ Therefore, an additional $28 \%$ or $11 \%$ of women with diagnoses of hypertension (depending on the cutoff used) would substantially increase the burden on health systems under stress, and could be justified only by the ability to identify women and babies at risk.

To our knowledge, this is the first study to report on the diagnostic properties of using revised blood pressure thresholds for the diagnosis of hypertension in pregnancy, and the first to report on outcomes by specific blood pressure thresholds for women in under-resourced settings.

Numerous studies have reported that women with antenatal elevated blood pressure or stage 1 hypertension by American College of Cardiology and American Heart Association criteria, as used in our analyses, have an increased risk of adverse pregnancy outcomes; ; $^{3,417-19}$ these included pre-eclampsia and other outcomes (eg, hospitalisation) not reported in our study. Many published RRs for adverse maternal and fetal or neonatal outcomes have been higher than in our study. It is possible that the relationship between blood pressure and adverse outcomes might be different in our study setting. Furthermore, we had fewer baseline characteristics with which to adjust our RRs compared with other studies, ${ }^{4,17-20}$ and we studied unselected pregnant women, not just those who were nulliparous ${ }^{21,22}$ or primarily nulliparous. ${ }^{23}$ Alternatively, the differences in RRs might have been due to methodology. Our data collection was prospective and blood pressure measurement was standardised, using a device validated for pregnancy and pre-eclampsia. ${ }^{11}$ By contrast, most published data have been retrospective and from large urban referral centres with blood pressure measurements from routine clinical care. ${ }^{4,17-20,23}$ One prospective study restricted blood pressure observations to women at less than 20 weeks of gestation, ${ }^{21,22}$ by contrast to our blood pressure measurements that were done throughout pregnancy, the majority of which were done after 20 weeks of gestation. One prospective study in South Africa reported that, among 1116 women, an additional $37 \cdot 1 \%$ would be classified as having abnormal blood pressure by the American College of Cardiology and American Heart Association criteria; although pregnancy outcomes were not reported separately for these women compared with those with stage 2 hypertension, ${ }^{24}$ it has been reported among pregnant teenagers in the same setting that eclampsia might follow antenatal blood pressure values of less than 140/90 mm Hg. ${ }^{25}$
Importantly, none of the aforementioned studies have reported the diagnostic test properties of various blood pressure categories to define abnormal blood pressure in pregnancy. As blood pressure measurement in pregnancy is a screening test, it should have high sensitivity for the adverse outcomes of interest, which can then lead to the established therapeutic care pathways for hypertension in pregnancy. However, blood pressure measurement is not sensitive, whether American College of Cardiology and American Heart Association categories are used (even elevated blood pressure) or an optimal blood pressure cutoff is estimated from the data and chosen. Perhaps this poor sensitivity is unsurprising, as the hypertensive disorders of pregnancy are one of three leading causes of adverse maternal outcomes (along with obstetric haemorrhage and sepsis), including spontaneous preterm birth, fetal growth restriction, and intrapartum complications for fetal or neonatal outcomes, ${ }^{26}$ and these other conditions could not be predicted by abnormal blood pressure. Nevertheless, although blood pressure measurement is regarded by WHO as good clinical practice that does not require evidence review, ${ }^{27}$ measurement is but one part of quality antenatal care and when normal, might provide minimal reassurance that adverse outcomes are less likely to occur.

The one blood pressure category that was useful in identifying women at increased risk of adverse outcomes (ie, maternal CNS outcomes and fetal or neonatal death) was severe stage 2 hypertension. This finding endorses severe stage 2 hypertension as a condition that should be avoided, as have previous data from well resourced settings, ${ }^{28}$ by contrast to regarding severe hypertension as a condition that can be treated if it occurs..$^{29}$

Our study has several strengths, including our large sample size, community-based recruitment of unselected pregnant women in south Asia and sub-Saharan Africa, and the standardisation of blood pressure readings across the sample, using a pregnancy-validated device. ${ }^{1{ }^{1}}$ These factors help to provide evidence for hypertension diagnostic thresholds that is applicable to a large set of pregnant women in these settings.

This study has several limitations. First, many women in Pakistan and Mozambique were enrolled after 20 weeks of gestation, consistent with timing of booking for antenatal care in these settings; therefore, a diagnosis of chronic hypertension could not be evaluated when blood pressure was measured after 20 weeks of gestation. Second, only basic maternal characteristics were available for our adjusted analyses. Third, many women did not have weekly blood pressure measurements from 36 weeks of gestation to delivery as specified in the CLIP protocol, so although the household survey and facility records ultimately informed diagnoses of hypertension, we did not have all relevant blood pressure values for this analysis, particularly from close to term. Women with stage 2 hypertension, whether severe or non-severe, were 
referred to facilities for antihypertensive treatment (about which we have no further information) and facility care; although not the focus of our analyses, such management is likely to have attenuated the relationship between stage 2 hypertension and outcomes and overestimated the strength of association between lower levels of blood pressure and adverse outcomes. Finally, we did not include in our analysis the outcome of preeclampsia given our community setting, and so could not evaluate this as an outcome with the revised hypertension definitions embedded in it.

Pregnant women in under-resourced settings with higher-than-normal blood pressure are at increased risk of adverse outcomes, due to the risks associated with blood pressure of 140/90 mm Hg or higher, particularly with severe stage 2 hypertension. No antenatal blood pressure threshold is sensitive with regards to the adverse maternal, fetal, or neonatal outcomes studied, including data-driven cutoffs. However, the occurrence of severe stage 2 hypertension ( $\geq 160 / 110 \mathrm{~mm} \mathrm{Hg}$ ) is associated with a substantially increased risk of adverse maternal CNS outcomes and fetal or neonatal death, particularly stillbirth, and should be treated as per international guidance. ${ }^{30}$ Antenatal care must aim to provide more than accurate blood pressure measurement to achieve the Countdown 2030 goals.

\section{Contributors}

PvD designed the concept of the CLIP trials. ZAB and LAM were the co-principal investigators of the overall CLIP trials project. MBB, ZAB, SSG, AAM, RNQ, KM, CS, and ES were co-principal investigators of the individual CLIP trials. AS and HN designed the blood pressure device used and assisted with its implementation and some analyses.

MV coordinated the CLIP trials. The analysis plan was conceived by JNB and JS. JNB did the data analysis and wrote the first draft of the manuscript with LAM. JNB and LAM accessed and verified the data. All authors had full access to all the data in the study, and read and approved the final version of the manuscript. JNB had access to all data and had responsibility for its integrity and the data analysis. The corresponding author had final responsibility for the decision to submit for publication.

Declaration of interests

LAM and PvD report grants from the Bill \& Melinda Gates Foundation, during the conduct of the study. All other authors declare no competing interests.

\section{Data sharing}

A data sharing statement for the CLIP trials is provided in the appendix (pp 294-95).

\section{Acknowledgments}

The CLIP trials were funded by the University of British Columbia, a grantee of the Gates Foundation (PRE-EMPT initiative, OPP1017337). We thank the Government of Mozambique, Province of Sindh, and Government of India for permission to integrate the CLIP trials into their health systems with in-kind support.

\section{References}

1 Scott G, Gillon TE, Pels A, von Dadelszen P, Magee LA. Guidelines similarities and dissimilarities: a systematic review of international clinical practice guidelines for pregnancy hypertension. Am J Obstet Gynecol 2020; 0: S0002-9378(20)30846-2.

2 Whelton PK, Carey RM, Aronow WS, et al. 2017 ACC/AHA/AAPA/ $\mathrm{ABC} / \mathrm{ACPM} / \mathrm{AGS} / \mathrm{APhA} / \mathrm{ASH} / \mathrm{ASPC} / \mathrm{NMA} / \mathrm{PCNA}$ guideline for the prevention, detection, evaluation, and management of high blood pressure in adults: executive summary: a report of the American College of Cardiology/American Heart Association task force on clinical practice guidelines. Hypertension 2018; 71: 1269-324.
3 Sisti G, Williams B. Body of evidence in favor of adopting $130 / 80 \mathrm{~mm} \mathrm{Hg}$ as new blood pressure cut-off for all the hypertensive disorders of pregnancy. Medicina (Kaunas) 2019; 55: 703.

4 Reddy M, Rolnik DL, Harris K, et al. Challenging the definition of hypertension in pregnancy: a retrospective cohort study. Am J Obstet Gynecol 2020; 222: 606.

5 Sevene E, Sharma S, Munguambe K, et al. Community-Level Interventions for Pre-eclampsia (CLIP) in Mozambique: a cluster randomised controlled trial. Pregnancy Hypertens 2020; 21: 96-105.

6 Qureshi RN, Sheikh S, Hoodbhoy Z, et al. Community-Level Interventions for Pre-eclampsia (CLIP) in Pakistan: a cluster randomised controlled trial. Pregnancy Hypertens 2020; 22: 109-18.

7 Bellad MB, Goudar SS, Mallapur AA, et al. Community Level Interventions for Pre-eclampsia (CLIP) in India: a cluster randomised controlled trial. Pregnancy Hypertens 2020; 21: 166-75.

8 von Dadelszen P, Bhutta ZA, Sharma S, et al. The CommunityLevel Interventions for Pre-eclampsia (CLIP) cluster randomised trials in Mozambique, Pakistan, and India: an individual participant-level meta-analysis. Lancet 2020; 396: 553-63.

9 Thaddeus S, Maine D. Too far to walk: maternal mortality in context. Soc Sci Med 1994; 38: 1091-110.

10 Lim J, Cloete G, Dunsmuir DT, et al. Usability and feasibility of PIERS on the move: an mHealth app for pre-eclampsia triage. JMIR Mhealth Uhealth 2015; 3: e37.

11 Nathan HL, de Greeff A, Hezelgrave NL, Chappell LC, Shennan AH. An accurate semiautomated oscillometric blood pressure device for use in pregnancy (including pre-eclampsia) in a low-income and middle-income country population: the Microlife 3AS1-2. Blood Press Monit 2015; 20: 52-55.

12 Brown MA, Magee LA, Kenny LC, et al. Hypertensive disorders of pregnancy: ISSHP classification, diagnosis, and management recommendations for international practice. Hypertension 2018; 72: 24-43.

13 Zou GY, Donner A. Extension of the modified Poisson regression model to prospective studies with correlated binary data. Stat Methods Med Res 2013; 22: 661-70.

14 Deeks JJ, Altman DG. Diagnostic tests 4: likelihood ratios. BMJ 2004; 329: 168-69.

15 R Core Team. R: a language and environment for statistical computing. Vienna: R Foundation for Statistical Computing, 2020.

16 Magee LA, Sharma S, Nathan HL, et al. The incidence of pregnancy hypertension in India, Pakistan, Mozambique, and Nigeria: a prospective population-level analysis. PLoS Med 2019; 16: e1002783.

17 Greenberg VR, Lundsberg LS, Culhane JF, et al. 143: Is stage 1 hypertension prior to 20 weeks gestation associated with adverse perinatal outcomes? Am J Obstet Gynecol 2020; 222: S106-07.

18 González-Valencia DP, Valero-Rubio SY, Fernando Grillo-Ardila C. Prehypertension as a risk factor for the development of perinatal complications: retrospective cohort study. Pregnancy Hypertens 2020; 21: 203-07.

19 Wu DD, Gao L, Huang O, et al. Increased adverse pregnancy outcomes associated with stage 1 hypertension in a low-risk cohort: evidence from 47874 cases. Hypertension 2020; 75: 772-80.

$20 \mathrm{Hu}$ J, Li Y, Zhang B, et al. Impact of the 2017 ACC/AHA guideline for high blood pressure on evaluating gestational hypertensionassociated risks for newborns and mothers. Circ Res 2019; 125: 184-94.

21 Hauspurg A, Sutton EF, Catov JM, Caritis SN. Aspirin effect on adverse pregnancy outcomes associated with stage 1 hypertension in a high-risk cohort. Hypertension 2018; 72: 202-07.

22 Sutton EF, Hauspurg A, Caritis SN, Powers RW, Catov JM. Materna outcomes associated with lower range stage 1 hypertension. Obstet Gynecol 2018; 132: 843-49.

23 Fukushima K, Morokuma S, Tsukimori K, Murata M, Wake N. Blood pressure measurements within the JNC7 pre-hypertensive range after 32 weeks of gestation are a risk factor for decreased fetal growth. Hypertens Res 2012; 35: 128-30.

24 Bengtson AM, Phillips TK, le Roux SM, et al. High blood pressure at entry into antenatal care and birth outcomes among a cohort of HIV-uninfected women and women living with HIV initiating antiretroviral therapy in South Africa. Pregnancy Hypertens 2021; 23: 79-86. 
Articles

25 Moodley J, Ngene NC. Maternal deaths due to eclampsia in teenagers: lessons from assessment of maternal deaths in South Africa. Afr J Prim Health Care Fam Med 2020; 12: e1-6.

26 Kassebaum NJ, Barber RM, Bhutta ZA, et al. Global, regional, and national levels of maternal mortality, 1990-2015: a systematic analysis for the Global Burden of Disease Study 2015. Lancet 2016; 388: $1775-812$

27 WHO. WHO recommendations on antenatal care for a positive pregnancy experience. May 3, 2017. https://www.who.int/ publications-detail-redirect/9789241549912 (accessed Jan 8, 2021).
28 Magee LA, von Dadelszen P, Singer J, et al. The CHIPS randomized controlled trial (Control of Hypertension In Pregnancy Study): is severe hypertension just an elevated blood pressure? Hypertension 2016; 68: 1153-59.

29 Magee LA, von Dadelszen P, Rey E, et al. Less-tight versus tight control of hypertension in pregnancy. N Engl J Med 2015; 372: 407-17.

30 WHO. WHO recommendations for prevention and treatment of pre-eclampsia and eclampsia. 2011. http://www.who.int/ reproductivehealth/publications/maternal_perinatal health/9789241548335/en/ (accessed Jan 8, 2021). 